\begin{tabular}{|c|c|c|}
\hline 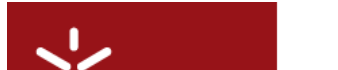 & CMAT & 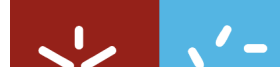 \\
\hline - Reprositónill & Centro de Matemática da Universidade do Minho & -0 \\
\hline \multirow[t]{2}{*}{ Universidade do Minho } & Campus de Gualtar $4710-057$ Braga Portugal & $\begin{array}{l}\text { Universidade do Minho } \\
\text { Escola de Ciências }\end{array}$ \\
\hline & www.cmat.uminho.pt & Centro de Matemática \\
\hline
\end{tabular}

\title{
A class of electromagnetic p-curl systems: blow-up and finite time extinction
}

\author{
Stanislav Antontsev ${ }^{a} \quad$ Fernando Miranda $^{b} \quad$ Lisa Santos ${ }^{b}$
}

${ }^{a}$ CMAF, Universidade de Lisboa, Portugal

${ }^{b}$ Departamento de Matemática e Aplicações and Centro de Matemática, Universidade do Minho, Portugal

\section{Information}

MSC:

35K55, 35Q61, 35B40, 35B44.

Keywords:

Electromagnetic problems, p-curl systems, blow-up, extinction in time.

\section{Original publication:}

Nonlinear Analysis 75, 3916-3929, 2012

DOI: $10.1016 /$ j.na.2012.02.011

www.elsevier.com/locate/na

\begin{abstract}
We study a class of $p$-curl systems arising in electromagnetism, for $\frac{6}{5}<p<\infty$, with nonlinear source or sink terms. Denoting by $\boldsymbol{h}$ the magnetic field, the source terms considered are of the form $\boldsymbol{h}\left(\int_{\Omega}|\boldsymbol{h}|^{2}\right)^{\frac{\sigma-2}{2}}$, with $\sigma \geq 1$. Existence of local or global solutions is proved depending on values of $\sigma$ and $p$. The blow-up of local solutions is also studied. The sink term is of the form $\boldsymbol{h}\left(\int_{\Omega}|\boldsymbol{h}|^{k}\right)^{-\lambda}$, with $k, \lambda>0$. Existence and finite time extinction of solutions are proved, for certain values of $k$ and $\lambda$.
\end{abstract}

\section{Introduction}

In the present paper, we study a class of p-curl systems arising in electromagnetism. We consider, as a starting point, the generalized Maxwell equations

$$
\begin{aligned}
\partial_{t} \boldsymbol{d}+\boldsymbol{j} & =\nabla \times \boldsymbol{h}, \\
\partial_{t} \boldsymbol{b}+\nabla \times \boldsymbol{e} & =\boldsymbol{f}, \\
\nabla \cdot \boldsymbol{d} & =q, \\
\nabla \cdot \boldsymbol{b} & =0,
\end{aligned}
$$

where $\boldsymbol{e}$ and $\boldsymbol{h}$ are the electric and magnetic fields, $\boldsymbol{d}$ and $\boldsymbol{b}$ the electric and magnetic inductions, $\boldsymbol{j}$ the total current density and $q$ the electric charge. We denote $\partial_{t}=\frac{\partial}{\partial t}, \nabla \times=$ curl, $\nabla \cdot=$ div.

As in [1], we adopt $\boldsymbol{b}=\mu \boldsymbol{h}$ as polarization law and $|\boldsymbol{j}|^{p-2} \boldsymbol{j}=\sigma \boldsymbol{e}$ as Ohm's law, where $\mu$ is the magnetic permeability and $\sigma$ the electric conductivity, and we neglect the term $\partial_{t} \boldsymbol{d}$. We assume, for simplicity, $\mu=$ $\sigma=1$.

Let $\Omega$ be a bounded open subset of $\mathbb{R}^{3}$ with Lipschitz boundary $\Gamma, T$ a positive real number, $Q_{T}=\Omega \times(0, T)$ and $\Sigma_{T}=\Gamma \times(0, T)$.

Considering the natural boundary conditions corresponding to a superconductive wall,

$$
\boldsymbol{h} \cdot \boldsymbol{n}=0 \quad \text { and } \quad \boldsymbol{e} \times \boldsymbol{n}=\mathbf{0} \text { on } \Sigma_{T}
$$


where $\boldsymbol{n}$ denotes the external unitary normal vector to the boundary $\Gamma$, and $\boldsymbol{h}(0)=\boldsymbol{h}_{0}$ as initial condition, where $\boldsymbol{h}_{0}$ is divergence free, we arrive at the system

$$
\begin{aligned}
& \partial_{t} \boldsymbol{h}+\nabla \times\left(|\nabla \times \boldsymbol{h}|^{p-2} \nabla \times \boldsymbol{h}\right)=\boldsymbol{f}(\boldsymbol{h}), \quad \nabla \cdot \boldsymbol{h}=0 \quad \text { in } Q_{T}, \\
& |\nabla \times \boldsymbol{h}|^{p-2} \nabla \times \boldsymbol{h} \times \boldsymbol{n}=\mathbf{0}, \quad \boldsymbol{h} \cdot \boldsymbol{n}=0 \quad \text { on } \Sigma_{T}, \\
& \boldsymbol{h}(\cdot, 0)=\boldsymbol{h}_{0} \quad \text { in } \Omega .
\end{aligned}
$$

For mathematical purposes we consider, in the first equation of (1a), nonlinear fields, sources or sinks, $\boldsymbol{f}(\boldsymbol{h})$, with special structure, verifying the compatibility conditions $\nabla \cdot \boldsymbol{f}(\boldsymbol{h})=0$ in $Q_{T}$ and $\boldsymbol{f}(\boldsymbol{h}) \cdot \boldsymbol{n}=0$ on $\Sigma_{T}$. The introduction of $\boldsymbol{f}(\boldsymbol{h})$ causes special qualitative properties of solutions, such as blow-up in a finite time (an explosion of the magnetic field) or the finite time extinction (disappearance of the magnetic field in finite time). We believe that these qualitative properties may be important to understand some physical processes or to motivate the creation of new numerical algorithms. In the considered cases we first prove theorems of existence of solutions and then we analyze their properties.

In Section 2, we introduce the functional framework and the weak formulation of problem (1).

In Section 3, considering the source term $\boldsymbol{f}(\boldsymbol{h})=\boldsymbol{h}\left(\int_{\Omega}|\boldsymbol{h}|^{2}\right)^{\frac{\sigma-2}{2}}$, we prove the existence of weak solution when $p>\frac{6}{5}$ and $\sigma \geq 1$. The solution is global if $\sigma \leq 2$ or $2<\sigma<p$ and is local otherwise. Assuming that the energy at the instant $t=0$ is nonpositive, the blow-up of local solutions is proved.

In Section 4, we consider the sink term $\boldsymbol{f}(\boldsymbol{h})=-\boldsymbol{h}\left(\int_{\Omega}|\boldsymbol{h}|^{k}\right)^{-\lambda}$, proving the existence of global weak solution for $p>\frac{6}{5}, 1 \leq k<p^{*}$ (critical Sobolev exponent) and for $\lambda$ positive and bounded from above, depending on $k$. In the particular case $k=2$ we prove uniqueness of solution for $0<\lambda \leq \frac{1}{2}$. The finite time extinction of solutions is proved for $\lambda>0$ and $0<k \leq p^{*}$ if $p<3$ and $k>0$ if $p \geq 3$. $5,6]$.

The proofs of the blow-up and finite time extinction of weak solutions use methods developed in $[2,3,4$,

\section{Weak formulation}

Before presenting the weak formulation of problem (1) we need to introduce the functional framework.

From now on $\Omega$ is a bounded simply connected domain with $\mathscr{C}^{1,1}$ boundary.

Spaces of vector-functions will be denoted by boldface symbols, following the standard notations for vectorfunctions.

Let us denote by $\boldsymbol{H}(\operatorname{div} 0, \Omega)$ the space of the divergence-free $\boldsymbol{L}^{2}(\Omega)$ functions endowed with the $\boldsymbol{L}^{2}$-norm.

We define the vector space

$$
\mathbb{W}^{p}(\Omega)=\left\{\boldsymbol{v} \in \boldsymbol{L}^{p}(\Omega): \nabla \times \boldsymbol{v} \in \boldsymbol{L}^{p}(\Omega), \nabla \cdot \boldsymbol{v}=0 \text { and } \boldsymbol{v} \cdot \boldsymbol{n}_{\left.\right|_{\Gamma}}=0\right\} .
$$

Remark 1. $\mathbb{W}^{p}(\Omega)$ is a closed subspace of $\boldsymbol{W}^{1, p}(\Omega)$. In $\mathbb{W}^{p}(\Omega)$, the semi-norm $\|\nabla \times \cdot\|_{\boldsymbol{L}^{p}(\Omega)}$ is a norm, equivalent to the $\boldsymbol{W}^{1, p}$-norm (for details, see $[7,8]$ ).

Let us also introduce the functional space

$$
\boldsymbol{X}_{p}\left(Q_{T}\right)=\left\{\boldsymbol{v} \in L^{p}\left(0, T ; \mathbb{W}^{p}(\Omega)\right): \partial_{t} \boldsymbol{v} \in L^{p^{\prime}}\left(0, T ; \mathbb{W}^{p}(\Omega)^{\prime}\right)\right\},
$$

with the norm $\|\boldsymbol{v}\|_{\boldsymbol{X}_{p}\left(Q_{T}\right)}=\|\boldsymbol{v}\|_{L^{p}\left(0, T ; \mathbb{W}^{p}(\Omega)\right)}+\left\|\partial_{t} \boldsymbol{v}\right\|_{L^{p^{\prime}\left(0, T ; \mathbb{W}^{p}(\Omega)^{\prime}\right)}}$.

With convenient assumptions on $\boldsymbol{f}$ and $\boldsymbol{h}_{0}$, we consider as weak formulation of problem (1), to find $\boldsymbol{h} \in \boldsymbol{X}_{p}\left(Q_{T}\right) \cap \mathscr{C}(0, T ; \boldsymbol{H}(\operatorname{div} 0, \Omega))$ satisfying, for a.e. $t \in(0, T)$,

$$
\begin{aligned}
& \int_{\Omega} \partial_{t} \boldsymbol{h}(t) \cdot \boldsymbol{\psi}+\int_{\Omega}|\nabla \times \boldsymbol{h}(t)|^{p-2} \nabla \times \boldsymbol{h}(t) \cdot \nabla \times \boldsymbol{\psi}=\int_{\Omega} \boldsymbol{f}(\boldsymbol{h}(t)) \cdot \boldsymbol{\psi}, \quad \forall \boldsymbol{\psi} \in \mathbb{W}^{p}(\Omega), \\
& \boldsymbol{h}(\cdot, 0)=\boldsymbol{h}_{0} \quad \text { in } \Omega,
\end{aligned}
$$

where the integral $\int_{\Omega} \partial_{t} \boldsymbol{h}(t) \cdot \boldsymbol{\psi}$ is interpreted in the duality $\mathbb{W}^{p}(\Omega)^{\prime}-\mathbb{W}^{p}(\Omega)$.

We present now some auxiliary lemmas. 
Lemma 2. There exists a positive constant $C$ such that, for $\boldsymbol{h} \in \mathbb{W}^{p}(\Omega)$,

$$
\|\boldsymbol{h}\|_{\boldsymbol{L}^{q}(\Omega)} \leq C\|\nabla \times \boldsymbol{h}\|_{\boldsymbol{L}^{p}(\Omega)},
$$

where for

$$
1 \leq p<3, q \leq \frac{3 p}{3-p} ; \quad p=3, q<\infty ; \quad p>3, q=\infty .
$$

Proof. This is an immediate consequence of Remark 1 and the Sobolev Embedding Theorem.

Lemma 3. Given $1 \leq k<2$, there exists a positive constant $C$ such that, for $\boldsymbol{h} \in \mathbb{W}^{p}(\Omega)$,

$$
\int_{\Omega}|\boldsymbol{h}|^{2} \leq C\left(\int_{\Omega}|\nabla \times \boldsymbol{h}|^{p}\right)^{\frac{2 \theta}{p}}\left(\int_{\Omega}|\boldsymbol{h}|^{k}\right)^{\frac{2(1-\theta)}{k}},
$$

where for

$$
p<3, \theta=\frac{\frac{1}{k}-\frac{1}{2}}{\frac{1}{k}-\frac{3-p}{3 p}} ; \quad p \geq 3, \theta \in(0,1] .
$$

Proof. By Remark 1 the $\mathbb{W}^{p}$-norm is equivalent to the $\boldsymbol{W}^{1, p}$-norm and so the conclusion follows from Lemma 3.3, p.297, of the Appendix of [2].

Lemma 4. Given $\boldsymbol{h} \in \mathbb{W}^{p}(\Omega)$, there exists a positive constant $C$ such that

$$
\int_{\Omega}|\boldsymbol{h}|^{k} \leq C\left(\int_{\Omega}|\nabla \times \boldsymbol{h}|^{p}\right)^{\frac{k \theta}{p}}\left(\int_{\Omega}|\boldsymbol{h}|^{2}\right)^{\frac{k(1-\theta)}{2}},
$$

where for

$$
p<3,0<k \leq \frac{3 p}{3-p} \text { and } \theta=\frac{\frac{1}{2}-\frac{1}{k}}{\frac{1}{2}-\frac{3-p}{3 p}} ; \quad p \geq 3, k>0 \text { and } \theta \in(0,1] .
$$

Proof. Use the same arguments of the proof of Lemma 3.

3 Blow-up: $\boldsymbol{f}(\boldsymbol{h})=\boldsymbol{h}\left(\int_{\Omega}|\boldsymbol{h}|^{2}\right)^{\frac{\sigma-2}{2}}$

In this section, for $\frac{6}{5}<p<\infty$, we prove the existence of global or local solution of problem (2), depending on the values of $\sigma \geq 1$. We also find sufficient conditions for the blow-up of solutions.

\subsection{Existence}

Considering $\boldsymbol{f}(\boldsymbol{h})=\boldsymbol{h}\left(\int_{\Omega}|\boldsymbol{h}|^{2}\right)^{\frac{\sigma-2}{2}}$ in the right-hand side of the first equation of (1a), where $\boldsymbol{f}(\boldsymbol{h})=\mathbf{0}$ whenever $\boldsymbol{h}=\mathbf{0}$, we prove the existence of solution of problem (2), with $p>\frac{6}{5}$ and $\sigma \geq 1$, using a fixed point argument.

For this purpose, given $\varphi$ defined in $Q_{T}$, we want to solve the problem

$$
\begin{aligned}
& \int_{\Omega} \partial_{t} \boldsymbol{h}_{\boldsymbol{\varphi}}(t) \cdot \boldsymbol{\psi}+\int_{\Omega}\left|\nabla \times \boldsymbol{h}_{\boldsymbol{\varphi}}(t)\right|^{p-2} \nabla \times \boldsymbol{h}_{\boldsymbol{\varphi}}(t) \cdot \nabla \times \boldsymbol{\psi} \\
&= \int_{\Omega} \boldsymbol{\varphi}(t) \cdot \boldsymbol{\psi}\left(\int_{\Omega}|\boldsymbol{\varphi}(t)|^{2}\right)^{\frac{\sigma-2}{2}}, \quad \forall \boldsymbol{\psi} \in \mathbb{W}^{p}(\Omega), \\
& \boldsymbol{h}_{\boldsymbol{\varphi}}(\cdot, 0)=\boldsymbol{h}_{0} \quad \text { in } \Omega .
\end{aligned}
$$


Proposition 5. Let us consider $\boldsymbol{\varphi} \in \mathscr{C}(0, T ; \boldsymbol{H}(\operatorname{div} 0, \Omega)), \boldsymbol{h}_{0} \in \boldsymbol{H}(\operatorname{div} 0, \Omega), \frac{6}{5} \leq p<\infty$ and $\sigma \geq 1$. Problem (5) has a unique solution $\boldsymbol{h}_{\varphi} \in \boldsymbol{X}_{p}\left(Q_{T}\right) \cap \mathscr{C}(0, T ; \boldsymbol{H}(\operatorname{div} 0, \Omega))$.

Proof. This result is an immediate consequence of Proposition 2 of [1]. In fact, for $\boldsymbol{\varphi} \in \mathscr{C}(0, T ; \boldsymbol{H}(\operatorname{div} 0, \Omega))$ we have $\boldsymbol{f}(\boldsymbol{\varphi}) \in L^{p^{\prime}}\left(0, T ; \boldsymbol{L}^{q^{\prime}}(\Omega)\right)$, where $q^{\prime}$ is the conjugate exponent of $q$ defined in Lemma 2 .

From now on we fix the notation $Q_{t}=\Omega \times(0, t)$ for a given $t \in(0, T)$.

In the following lemmas we will present some a priori estimates to the solutions $\boldsymbol{h}_{\boldsymbol{\varphi}}$ of problem (5).

Lemma 6. Given $\varphi \in \mathscr{C}(0, T ; \boldsymbol{H}(\operatorname{div} 0, \Omega)), \boldsymbol{h}_{0} \in \boldsymbol{H}(\operatorname{div} 0, \Omega), \frac{6}{5} \leq p<\infty$ and $\sigma \geq 1$, there exists a constant $C$ depending on $T,|\Omega|,\|\boldsymbol{\varphi}\|_{L^{\infty}\left(0, T ; \boldsymbol{L}^{2}(\Omega)\right)}$ and $\left\|\boldsymbol{h}_{0}\right\|_{\boldsymbol{L}^{2}(\Omega)}$ such that

$$
\begin{aligned}
& \left\|\boldsymbol{h}_{\boldsymbol{\varphi}}\right\|_{L^{\infty}\left(0, T ; \boldsymbol{L}^{2}(\Omega)\right)} \leq C, \\
& \left\|\nabla \times \boldsymbol{h}_{\boldsymbol{\varphi}}\right\|_{L^{p}\left(Q_{T}\right)} \leq C .
\end{aligned}
$$

Proof. Using $\boldsymbol{h}_{\varphi}$ as a test function in equation (5a) and integrating between 0 and $t$, we obtain

$$
\int_{0}^{t} \int_{\Omega} \partial_{t} \boldsymbol{h}_{\varphi} \cdot \boldsymbol{h}_{\varphi}+\int_{Q_{t}}\left|\nabla \times \boldsymbol{h}_{\varphi}\right|^{p}=\int_{Q_{t}} \boldsymbol{\varphi} \cdot \boldsymbol{h}_{\boldsymbol{\varphi}}\left(\int_{\Omega}|\boldsymbol{\varphi}|^{2}\right)^{\frac{\sigma-2}{2}}
$$

and so we can write, using the Hölder inequality,

$$
\frac{1}{2} \int_{\Omega}\left|\boldsymbol{h}_{\boldsymbol{\varphi}}(t)\right|^{2}+\int_{Q_{t}}\left|\nabla \times \boldsymbol{h}_{\boldsymbol{\varphi}}\right|^{p} \leq \int_{0}^{t}\|\boldsymbol{\varphi}(\tau)\|_{L^{2}(\Omega)}^{\sigma-1}\left\|\boldsymbol{h}_{\boldsymbol{\varphi}}(\tau)\right\|_{\boldsymbol{L}^{2}(\Omega)}+\frac{1}{2} \int_{\Omega}\left|\boldsymbol{h}_{0}\right|^{2} .
$$

Applying now the Young inequality and Remark 1 of [1], there exists a constant $\tilde{C}$, depending on $\Omega$ and $p$ such that

$$
\int_{\Omega}\left|\boldsymbol{h}_{\boldsymbol{\varphi}}(t)\right|^{2}+\int_{Q_{t}}\left|\nabla \times \boldsymbol{h}_{\boldsymbol{\varphi}}\right|^{p} \leq \tilde{C} \int_{0}^{t}\|\boldsymbol{\varphi}(\tau)\|_{\boldsymbol{L}^{2}(\Omega)}^{p^{\prime}(\sigma-1)}+\int_{\Omega}\left|\boldsymbol{h}_{0}\right|^{2}
$$

and so

$$
\left\|\boldsymbol{h}_{\boldsymbol{\varphi}}\right\|_{L^{\infty}\left(0, T ; \boldsymbol{L}^{2}(\Omega)\right)}^{2}+\left\|\nabla \times \boldsymbol{h}_{\boldsymbol{\varphi}}\right\|_{\boldsymbol{L}^{p}\left(Q_{T}\right)}^{p} \leq \tilde{C} T\|\boldsymbol{\varphi}\|_{L^{\infty}\left(0, T ; \boldsymbol{L}^{2}(\Omega)\right)}^{p^{\prime}(\sigma-1)}+\left\|\boldsymbol{h}_{0}\right\|_{\boldsymbol{L}^{2}(\Omega)}^{2} .
$$

which proves (6) and (7).

Lemma 7. Given $\varphi \in \mathscr{C}(0, T ; \boldsymbol{H}(\operatorname{div} 0, \Omega)), \boldsymbol{h}_{0} \in \mathbb{W}^{p}(\Omega), \frac{6}{5} \leq p<\infty$ and $\sigma \geq 1$, there exists a constant $C$ depending on $T,\|\boldsymbol{\varphi}\|_{L^{\infty}\left(0, T ; \boldsymbol{L}^{2}(\Omega)\right)}$ and $\left\|\boldsymbol{h}_{0}\right\|_{\mathbb{W}^{p}(\Omega)}$ such that

$$
\begin{aligned}
& \left\|\partial_{t} \boldsymbol{h}_{\boldsymbol{\varphi}}\right\|_{\boldsymbol{L}^{2}\left(Q_{T}\right)} \leq C, \\
& \left\|\nabla \times \boldsymbol{h}_{\boldsymbol{\varphi}}\right\|_{L^{\infty}\left(0, T ; \boldsymbol{L}^{p}(\Omega)\right)} \leq C .
\end{aligned}
$$

Proof. To obtain these estimates we need to introduce Galerkin approximations. Let $\left(\boldsymbol{\psi}_{n}\right)_{n}$ be a topological basis of $\mathbb{W}^{p}(\Omega)$ and denote by $\left\langle\boldsymbol{\psi}_{1}, \ldots, \boldsymbol{\psi}_{m}\right\rangle$ the subspace generated by $\left\{\boldsymbol{\psi}_{1}, \ldots, \boldsymbol{\psi}_{m}\right\}$. Consider a family of approximated problems in finite dimensions: to find

$$
\boldsymbol{h}_{m}(t)=\sum_{i=1}^{m} \lambda_{i}^{m}(t) \boldsymbol{\psi}_{i}
$$

verifying the following $m$ ordinary differential equations

$$
\begin{aligned}
& \int_{\Omega} \partial_{t} \boldsymbol{h}_{m}(t) \cdot \boldsymbol{\psi}_{i}+\int_{\Omega}\left|\nabla \times \boldsymbol{h}_{m}(t)\right|^{p-2} \nabla \times \boldsymbol{h}_{m}(t) \cdot \nabla \times \boldsymbol{\psi}_{i} \\
&= \int_{\Omega} \boldsymbol{\varphi}(t) \cdot \boldsymbol{\psi}_{i}\left(\int_{\Omega}|\boldsymbol{\varphi}(t)|^{2}\right)^{\frac{\sigma-2}{2}}, i=1, \ldots, m, \\
& \boldsymbol{h}_{m}(\cdot, 0)=\boldsymbol{h}_{m, 0} \quad \text { in } \Omega,
\end{aligned}
$$


where $\boldsymbol{h}_{0, m} \in\left\langle\boldsymbol{\psi}_{1}, \ldots, \boldsymbol{\psi}_{m}\right\rangle$ is such that $\boldsymbol{h}_{0, m} \underset{m}{\longrightarrow} \boldsymbol{h}_{0}$ in $\mathbb{W}^{p}(\Omega)$.

Observe that this system has a unique solution $\boldsymbol{h}_{m} \in \mathscr{C}^{1}\left(0, T ;\left\langle\boldsymbol{\psi}_{1}, \ldots, \boldsymbol{\psi}_{m}\right\rangle\right)$ and is equivalent to

$$
\begin{aligned}
& \int_{\Omega} \partial_{t} \boldsymbol{h}_{m}(t) \cdot \boldsymbol{\psi}(t)+\int_{\Omega}\left|\nabla \times \boldsymbol{h}_{m}(t)\right|^{p-2} \nabla \times \boldsymbol{h}_{m}(t) \cdot \nabla \times \boldsymbol{\psi}(t) \\
& =\int_{\Omega} \boldsymbol{\varphi}(t) \cdot \boldsymbol{\psi}(t)\left(\int_{\Omega}|\boldsymbol{\varphi}(t)|^{2}\right)^{\frac{\sigma-2}{2}}, \forall \boldsymbol{\psi} \in \mathscr{C}\left(0, T ;\left\langle\boldsymbol{\psi}_{1}, \ldots, \boldsymbol{\psi}_{m}\right\rangle\right), \\
& \boldsymbol{h}_{m}(\cdot, 0)=\boldsymbol{h}_{m, 0} \quad \text { in } \Omega .
\end{aligned}
$$

Using $\boldsymbol{h}_{m}$ as test functions in (12) and integrating in $(0, t)$ we obtain, using Hölder and Young inequalities,

$$
\int_{\Omega}\left|\boldsymbol{h}_{m}(t)\right|^{2}+2 \int_{Q_{t}}\left|\nabla \times \boldsymbol{h}_{m}\right|^{p} \leq \int_{0}^{t}\left(\int_{\Omega}|\boldsymbol{\varphi}|^{2}\right)^{\sigma-1}+\int_{Q_{t}}\left|\boldsymbol{h}_{m}\right|^{2}+\int_{\Omega}\left|\boldsymbol{h}_{0, m}\right|^{2} .
$$

Applying inequality (3), with $q=2$, we obtain the uniform boundedness of $\left(\boldsymbol{h}_{m}\right)_{m}$ in $L^{\infty}\left(0, T ; \boldsymbol{L}^{2}(\Omega)\right)$ and of $\left(\nabla \times \boldsymbol{h}_{m}\right)_{m}$ in $\boldsymbol{L}^{p}\left(Q_{T}\right)$ and so, since by Proposition 5 the solution $\boldsymbol{h}_{\varphi}$ is unique,

$$
\begin{aligned}
& \boldsymbol{h}_{m} \longrightarrow \boldsymbol{h}_{\boldsymbol{\varphi}} \text { in } L^{\infty}\left(0, T ; \boldsymbol{L}^{2}(\Omega)\right) \text { - weak*, } \\
& \left.\nabla \times \boldsymbol{h}_{m} \longrightarrow \nabla \times \boldsymbol{h}_{\boldsymbol{\varphi}} \text { in } \boldsymbol{L}^{p}\left(Q_{T}\right)\right) \text { - weak } *
\end{aligned}
$$

Using now $\partial_{t} \boldsymbol{h}_{m}$ as test functions in (12) and integrating in $(0, t)$ we obtain

$$
\int_{0}^{t} \int_{\Omega}\left|\partial_{t} \boldsymbol{h}_{m}\right|^{2}+\frac{1}{p} \int_{0}^{t} \int_{\Omega} \partial_{t}\left|\nabla \times \boldsymbol{h}_{m}\right|^{p} \leq \int_{0}^{t} \int_{\Omega}|\boldsymbol{\varphi}|\left|\partial_{t} \boldsymbol{h}_{m}\right|\left(\int_{\Omega}|\boldsymbol{\varphi}|^{2}\right)^{\frac{\sigma-2}{2}},
$$

and the same inequality is verified by $\boldsymbol{h}_{\boldsymbol{\varphi}}$. Applying Hölder and Young inequalities we obtain

$$
\frac{1}{2} \int_{0}^{t}\left\|\partial_{t} \boldsymbol{h}_{\boldsymbol{\varphi}}\right\|_{\boldsymbol{L}^{2}(\Omega)}^{2}+\frac{1}{p}\left\|\nabla \times \boldsymbol{h}_{\boldsymbol{\varphi}}(t)\right\|_{\boldsymbol{L}^{p}(\Omega)}^{p} \leq \frac{1}{2} T\|\boldsymbol{\varphi}\|_{L^{\infty}\left(0, T ; \boldsymbol{L}^{2}(\Omega)\right)}^{2(\sigma-1)}+\frac{1}{p}\left\|\nabla \times \boldsymbol{h}_{0}\right\|_{\boldsymbol{L}^{p}(\Omega)}^{p}
$$

and so the estimates (10) and (11) follow.

Proposition 8. Given $\boldsymbol{h}_{0} \in \mathbb{W}^{p}(\Omega), \frac{6}{5} \leq p<\infty$ and $\sigma \geq 1$, the function $S$ that maps $\varphi \in \mathscr{C}([0, T] ; \boldsymbol{H}(\operatorname{div} 0, \Omega))$ to $\boldsymbol{h}_{\boldsymbol{\varphi}} \in \boldsymbol{X}_{p}\left(Q_{T}\right)$, solution of problem (5), is continuous.

Proof. Let $\left(\boldsymbol{\varphi}_{n}\right)_{n}$ be a sequence convergent to $\varphi$ in $\mathscr{C}([0, T] ; \boldsymbol{H}(\operatorname{div} 0, \Omega))$. In order to prove that $S$ is continuous we are going to prove that $S\left(\boldsymbol{\varphi}_{n}\right)=\boldsymbol{h}_{\boldsymbol{\varphi}_{n}}$ converges to $S(\boldsymbol{\varphi})=\boldsymbol{h}_{\boldsymbol{\varphi}}$ in $L^{p}\left(0, T ; \mathbb{W}^{p}(\Omega)\right)$ and $\partial_{t} \boldsymbol{h}_{\boldsymbol{\varphi}_{n}}$ converges to $\partial_{t} \boldsymbol{h}_{\boldsymbol{\varphi}}$ in $L^{p^{\prime}}\left(0, T ; \mathbb{W}^{p}(\Omega)^{\prime}\right)$.

Recall that (see, for instance, [9]) there exists a positive constant $C_{p}$ such that, for all $\boldsymbol{\xi}, \boldsymbol{\eta} \in \mathbb{R}^{3}$, if $p \geq 2$, we have

$$
\left(|\boldsymbol{\xi}|^{p-2} \boldsymbol{\xi}-|\boldsymbol{\eta}|^{p-2} \boldsymbol{\eta}\right) \cdot(\boldsymbol{\xi}-\boldsymbol{\eta}) \geq C_{p}|\boldsymbol{\xi}-\boldsymbol{\eta}|^{p}
$$

and, if $1<p<2$,

$$
\left(|\boldsymbol{\xi}|^{p-2} \boldsymbol{\xi}-|\boldsymbol{\eta}|^{p-2} \boldsymbol{\eta}\right) \cdot(\boldsymbol{\xi}-\boldsymbol{\eta}) \geq C_{p}(|\boldsymbol{\xi}|+|\boldsymbol{\eta}|)^{p-2}|\boldsymbol{\xi}-\boldsymbol{\eta}|^{2} .
$$

We obtain the equation

$$
\begin{aligned}
& \int_{0}^{t} \int_{\Omega}\left(\partial_{t} \boldsymbol{h}_{\boldsymbol{\varphi}_{n}}-\partial_{t} \boldsymbol{h}_{\boldsymbol{\varphi}}\right) \cdot\left(\boldsymbol{h}_{\boldsymbol{\varphi}_{n}}-\boldsymbol{h}_{\boldsymbol{\varphi}}\right) \\
&+\int_{Q_{t}}\left(\left|\nabla \times \boldsymbol{h}_{\varphi_{n}}\right|^{p-2} \nabla \times \boldsymbol{h}_{\boldsymbol{\varphi}_{n}}-\left|\nabla \times \boldsymbol{h}_{\boldsymbol{\varphi}}\right|^{p-2} \nabla \times \boldsymbol{h}_{\boldsymbol{\varphi}}\right) \cdot \nabla \times\left(\boldsymbol{h}_{\boldsymbol{\varphi}_{n}}-\boldsymbol{h}_{\boldsymbol{\varphi}}\right) \\
& \quad=\int_{Q_{t}}\left(\boldsymbol{f}\left(\boldsymbol{\varphi}_{n}\right)-\boldsymbol{f}(\boldsymbol{\varphi})\right) \cdot\left(\boldsymbol{h}_{\boldsymbol{\varphi}_{n}}-\boldsymbol{h}_{\boldsymbol{\varphi}}\right),
\end{aligned}
$$


using $\boldsymbol{h}_{\boldsymbol{\varphi}_{n}}-\boldsymbol{h}_{\boldsymbol{\varphi}}$ as a test function in problem (2a) both for $\boldsymbol{h}_{\boldsymbol{\varphi}_{n}}$ and $\boldsymbol{h}_{\boldsymbol{\varphi}}$.

- The case $p \geq 2$

$$
\begin{aligned}
\frac{1}{2} \int_{\Omega}\left|\boldsymbol{h}_{\boldsymbol{\varphi}_{n}}(t)-\boldsymbol{h}_{\boldsymbol{\varphi}}(t)\right|^{2}+C_{p} \int_{Q_{t}} \mid \nabla \times & \left.\left(\boldsymbol{h}_{\boldsymbol{\varphi}_{n}}-\boldsymbol{h}_{\boldsymbol{\varphi}}\right)\right|^{p} \\
& \quad \leq C_{1} \int_{Q_{T}}\left|\boldsymbol{f}\left(\boldsymbol{\varphi}_{n}\right)-\boldsymbol{f}(\boldsymbol{\varphi})\right|^{2}+\frac{1}{2}\left\|\boldsymbol{h}_{\boldsymbol{\varphi}_{n}}-\boldsymbol{h}_{\boldsymbol{\varphi}}\right\|_{L^{\infty}\left(0, T ; \boldsymbol{L}^{2}(\Omega)\right)}^{2},
\end{aligned}
$$

and so

$$
\left\|\nabla \times\left(\boldsymbol{h}_{\boldsymbol{\varphi}_{n}}-\boldsymbol{h}_{\boldsymbol{\varphi}}\right)\right\|_{\boldsymbol{L}^{p}\left(Q_{T}\right)}^{p} \leq C \int_{Q_{T}}\left|\boldsymbol{f}\left(\boldsymbol{\varphi}_{n}\right)-\boldsymbol{f}(\boldsymbol{\varphi})\right|^{2} .
$$

- The case $\frac{6}{5} \leq p<2$

$$
\begin{aligned}
& \frac{1}{2} \int_{\Omega}\left|\boldsymbol{h}_{\boldsymbol{\varphi}_{n}}(t)-\boldsymbol{h}_{\boldsymbol{\varphi}}(t)\right|^{2} \\
& \quad+C_{p} \int_{Q_{t}}\left(\left|\nabla \times \boldsymbol{h}_{\boldsymbol{\varphi}_{n}}\right|+\left|\nabla \times \boldsymbol{h}_{\boldsymbol{\varphi}}\right|\right)^{p-2}\left|\nabla \times\left(\boldsymbol{h}_{\boldsymbol{\varphi}_{n}}-\boldsymbol{h}_{\boldsymbol{\varphi}}\right)\right|^{2} \\
& \quad \leq C_{1} \int_{Q_{T}}\left|\boldsymbol{f}\left(\boldsymbol{\varphi}_{n}\right)-\boldsymbol{f}(\boldsymbol{\varphi})\right|^{2}+\frac{1}{2}\left\|\boldsymbol{h}_{\boldsymbol{\varphi}_{n}}-\boldsymbol{h}_{\boldsymbol{\varphi}}\right\|_{L^{\infty}\left(0, T ; \boldsymbol{L}^{2}(\Omega)\right)}^{2}
\end{aligned}
$$

Applying the reverse Hölder inequality with $0<r=\frac{p}{2}$ and $r^{\prime}=\frac{p}{p-2}$ we get

$$
\begin{aligned}
\int_{Q_{t}}\left(\left|\nabla \times \boldsymbol{h}_{\boldsymbol{\varphi}_{n}}\right|+\left|\nabla \times \boldsymbol{h}_{\boldsymbol{\varphi}}\right|\right)^{p-2} \mid \nabla & \times\left.\left(\boldsymbol{h}_{\boldsymbol{\varphi}_{n}}-\boldsymbol{h}_{\boldsymbol{\varphi}}\right)\right|^{2} \\
& \geq\left(\int_{Q_{t}} \nabla \times\left.\left(\boldsymbol{h}_{\boldsymbol{\varphi}_{n}}-\boldsymbol{h}_{\boldsymbol{\varphi}}\right)\right|^{p}\right)^{\frac{2}{p}}\left(\int_{Q_{t}}\left(\left|\nabla \times \boldsymbol{h}_{\boldsymbol{\varphi}_{n}}\right|+\left|\nabla \times \boldsymbol{h}_{\boldsymbol{\varphi}}\right|\right)^{p}\right)^{\frac{p-2}{p}} .
\end{aligned}
$$

Using the last two inequalities we obtain

$$
\left\|\nabla \times\left(\boldsymbol{h}_{\boldsymbol{\varphi}_{n}}-\boldsymbol{h}_{\boldsymbol{\varphi}}\right)\right\|_{\boldsymbol{L}^{p}\left(Q_{T}\right)}^{2} \leq C_{2} \int_{Q_{T}}\left|\boldsymbol{f}\left(\boldsymbol{\varphi}_{n}\right)-\boldsymbol{f}(\boldsymbol{\varphi})\right|^{2}\left(\int_{Q_{t}}\left(\left|\nabla \times \boldsymbol{h}_{\boldsymbol{\varphi}_{n}}\right|+\left|\nabla \times \boldsymbol{h}_{\boldsymbol{\varphi}}\right|\right)^{p}\right)^{\frac{2-p}{p}} .
$$

By inequality (7) and because $\left(\boldsymbol{\varphi}_{n}\right)_{n}$ converges to $\varphi$ in $L^{\infty}\left(0, T ; \boldsymbol{L}^{2}(\Omega)\right)$, the term

$$
\left(\int_{Q_{t}}\left(\left|\nabla \times \boldsymbol{h}_{\varphi_{n}}\right|+\left|\nabla \times \boldsymbol{h}_{\boldsymbol{\varphi}}\right|\right)^{p}\right)^{\frac{2-p}{p}}
$$

is bounded independently of $n$.

In both cases we proved that

$$
\left\|\nabla \times\left(\boldsymbol{h}_{\boldsymbol{\varphi}_{n}}-\boldsymbol{h}_{\boldsymbol{\varphi}}\right)\right\|_{\boldsymbol{L}^{p}\left(Q_{T}\right)}^{2 \vee p} \leq C \int_{Q_{T}}\left|\boldsymbol{f}\left(\boldsymbol{\varphi}_{n}\right)-\boldsymbol{f}(\boldsymbol{\varphi})\right|^{2}, \text { where } 2 \vee p=\max \{2, p\} .
$$

But, by Lebesgue Theorem, $\int_{Q_{T}}\left|\boldsymbol{f}\left(\boldsymbol{\varphi}_{n}\right)-\boldsymbol{f}(\boldsymbol{\varphi})\right|^{2} \underset{n}{\longrightarrow} 0$ and so $\left(\boldsymbol{h}_{\boldsymbol{\varphi}_{n}}\right)_{n}$ tends to $\boldsymbol{h}_{\boldsymbol{\varphi}}$ in $L^{p}\left(0, T ; \mathbb{W}^{p}(\Omega)\right)$. In what follows $\langle\cdot, \cdot\rangle$ denotes the duality paring between $\mathbb{W}^{p}(\Omega)^{\prime}-\mathbb{W}^{p}(\Omega)$.

$$
\begin{array}{r}
\int_{0}^{T}\left\langle\partial_{t} \boldsymbol{h}_{\boldsymbol{\varphi}_{n}}(t)-\partial_{t} \boldsymbol{h}_{\boldsymbol{\varphi}}(t), \boldsymbol{\psi}(t)\right\rangle+\int_{Q_{T}}\left(\left|\nabla \times \boldsymbol{h}_{\boldsymbol{\varphi}_{n}}\right|^{p-2} \nabla \times \boldsymbol{h}_{\boldsymbol{\varphi}_{n}}-\left|\nabla \times \boldsymbol{h}_{\boldsymbol{\varphi}}\right|^{p-2} \nabla \times \boldsymbol{h}_{\boldsymbol{\varphi}}\right) \cdot \nabla \times \boldsymbol{\psi} \\
=\int_{Q_{T}}\left(\boldsymbol{f}\left(\boldsymbol{\varphi}_{n}\right)-\boldsymbol{f}(\boldsymbol{\varphi})\right) \cdot \boldsymbol{\psi}
\end{array}
$$


and so

$$
\begin{aligned}
\int_{0}^{T}\left\langle\partial_{t} \boldsymbol{h}_{\boldsymbol{\varphi}_{n}}\right. & \left.(t)-\partial_{t} \boldsymbol{h}_{\boldsymbol{\varphi}}(t), \boldsymbol{\psi}(t)\right\rangle \\
& \leq \int_{Q_{T}}|| \nabla \times\left.\boldsymbol{h}_{\boldsymbol{\varphi}_{n}}\right|^{p-2} \nabla \times \boldsymbol{h}_{\boldsymbol{\varphi}_{n}}-\left|\nabla \times \boldsymbol{h}_{\boldsymbol{\varphi}}\right|^{p-2} \nabla \times \boldsymbol{h}_{\boldsymbol{\varphi}}|| \nabla \times \boldsymbol{\psi}\left|+\int_{Q_{T}}\right| \boldsymbol{f}\left(\boldsymbol{\varphi}_{n}\right)-\boldsymbol{f}(\boldsymbol{\varphi})|| \boldsymbol{\psi} \mid .
\end{aligned}
$$

Applying Hölder's inequality and recalling that $L^{p}\left(0, T ; \mathbb{W}^{p}(\Omega)\right) \subseteq L^{p}\left(0, T ; \boldsymbol{L}^{q}(\Omega)\right)$, where $q^{\prime}$ is the conjugate exponent of $q$ defined in Lemma 2, we have

$$
\begin{aligned}
& \left\|\partial_{t} \boldsymbol{h}_{\boldsymbol{\varphi}_{n}}-\partial_{t} \boldsymbol{h}_{\boldsymbol{\varphi}}\right\|_{L^{p^{\prime}}\left(0, T ; \mathbb{W}^{p}(\Omega)^{\prime}\right)}=\sup _{\|\boldsymbol{\psi}\|_{L^{p}\left(0, T ; \mathbb{W}^{p}(\Omega)\right)} \leq 1} \int_{Q_{T}}\left(\partial_{t} \boldsymbol{h}_{\boldsymbol{\varphi}_{n}}-\partial_{t} \boldsymbol{h}_{\boldsymbol{\varphi}}\right) \cdot \boldsymbol{\psi} \\
& \quad \leq\left\|\left|\nabla \times \boldsymbol{h}_{\boldsymbol{\varphi}_{n}}\right|^{p-2} \nabla \times \boldsymbol{h}_{\boldsymbol{\varphi}_{n}}-\left|\nabla \times \boldsymbol{h}_{\boldsymbol{\varphi}}\right|^{p-2} \nabla \times \boldsymbol{h}_{\boldsymbol{\varphi}}\right\|_{\boldsymbol{L}^{p^{\prime}}\left(Q_{T}\right)}+C_{1}\left\|\boldsymbol{f}\left(\boldsymbol{\varphi}_{n}\right)-\boldsymbol{f}(\boldsymbol{\varphi})\right\|_{L^{p^{\prime}}\left(0, T ; \boldsymbol{L}^{q^{\prime}}(\Omega)\right)} .
\end{aligned}
$$

From the inequality

$$
\left\|\left|\nabla \times \boldsymbol{h}_{\boldsymbol{\varphi}_{n}}\right|^{p-2} \nabla \times \boldsymbol{h}_{\boldsymbol{\varphi}_{n}}-\left|\nabla \times \boldsymbol{h}_{\boldsymbol{\varphi}}\right|^{p-2} \nabla \times \boldsymbol{h}_{\boldsymbol{\varphi}}\right\|_{\boldsymbol{L}^{p^{\prime}}\left(Q_{T}\right)}^{p^{\prime}} \leq \begin{cases}C\left\|\nabla \times\left(\boldsymbol{h}_{\boldsymbol{\varphi}_{n}}-\boldsymbol{h}_{\boldsymbol{\varphi}}\right)\right\|_{\boldsymbol{L}_{p}^{p}\left(Q_{T}\right)}^{p}, & \text { if } p \leq 2, \\ C\left\|\nabla \times\left(\boldsymbol{h}_{\boldsymbol{\varphi}_{n}}-\boldsymbol{h}_{\boldsymbol{\varphi}}\right)\right\|_{\boldsymbol{L}^{p}\left(Q_{T}\right)}^{\frac{p-1}{p}}, & \text { if } p>2,\end{cases}
$$

as we proved that $\nabla \times \boldsymbol{h}_{\boldsymbol{\varphi}_{n}} \underset{n}{\longrightarrow} \nabla \times \boldsymbol{h}_{\boldsymbol{\varphi}}$ in $\boldsymbol{L}^{p}\left(Q_{T}\right)$, the term

$$
\left\|\left|\nabla \times \boldsymbol{h}_{\boldsymbol{\varphi}_{n}}\right|^{p-2} \nabla \times \boldsymbol{h}_{\boldsymbol{\varphi}_{n}}-\left|\nabla \times \boldsymbol{h}_{\boldsymbol{\varphi}}\right|^{p-2} \nabla \times \boldsymbol{h}_{\boldsymbol{\varphi}}\right\|_{\boldsymbol{L}^{p^{\prime}\left(Q_{T}\right)}}^{p^{\prime}} \underset{n}{\longrightarrow} 0 .
$$

On the other hand, as $L^{\infty}\left(0, T ; \boldsymbol{L}^{2}(\Omega)\right) \subseteq L^{p^{\prime}}\left(0, T ; \boldsymbol{L}^{q^{\prime}}(\Omega)\right)$, the term

$$
\left\|\boldsymbol{f}\left(\boldsymbol{\varphi}_{n}\right)-\boldsymbol{f}(\boldsymbol{\varphi})\right\|_{L^{p^{\prime}}\left(0, T ; \boldsymbol{L}^{q^{\prime}}(\Omega)\right)} \underset{n}{\longrightarrow} 0 .
$$

So we conclude that $\partial_{t} \boldsymbol{h}_{\boldsymbol{\varphi}_{n}} \underset{n}{\longrightarrow} \partial_{t} \boldsymbol{h}_{\boldsymbol{\varphi}}$ in $L^{p^{\prime}}\left(0, T ; \mathbb{W}^{p}(\Omega)^{\prime}\right)$.

Proposition 9. Given $\boldsymbol{h}_{0} \in \mathbb{W}^{p}(\Omega), \frac{6}{5}<p<\infty$ and $\sigma \geq 1$, the function $\tilde{S}=i \circ S$, where $i$ is the inclusion of $\boldsymbol{X}_{p}\left(Q_{T}\right)$ into $\mathscr{C}([0, T] ; \boldsymbol{H}(\operatorname{div} 0, \Omega))$, is compact.

Proof. Let $F_{R}=\left\{\tilde{S}(\boldsymbol{\varphi}):\|\boldsymbol{\varphi}\|_{L^{\infty}\left(0, T ; \boldsymbol{L}^{2}(\Omega)\right)} \leq R\right\}$. Recalling that $\tilde{S}(\boldsymbol{\varphi})=\boldsymbol{h}_{\boldsymbol{\varphi}}$, by the estimate $(11) F_{R}$ is bounded in $L^{\infty}\left(0, T ; \mathbb{W}^{p}(\Omega)\right)$.

Observing that

$$
\int_{\Omega}\left|\boldsymbol{h}_{\varphi}(t+\delta)-\boldsymbol{h}_{\boldsymbol{\varphi}}(t)\right|^{2}=\int_{\Omega}\left|\int_{t}^{t+\delta} \partial_{t} \boldsymbol{h}_{\boldsymbol{\varphi}}(\tau)\right|^{2} \leq \delta \int_{\Omega} \int_{t}^{t+\delta}\left|\partial_{t} \boldsymbol{h}_{\varphi}(\tau)\right|^{2} \leq \delta\left\|\partial_{t} \boldsymbol{h}_{\varphi}\right\|_{\boldsymbol{L}^{2}\left(Q_{T}\right)}^{2}
$$

and, as $\mathbb{W}^{p}(\Omega)$ is compactly included in $\boldsymbol{L}^{2}(\Omega)$ (and also in $\boldsymbol{H}(\operatorname{div} 0, \Omega)$ ), using Theorem 5 of [10] with $X=\mathbb{W}^{p}(\Omega)$ and $B=Y=\boldsymbol{H}(\operatorname{div} 0, \Omega)$, the conclusion follows.

Theorem 10. For $\boldsymbol{h}_{0} \in \mathbb{W}^{p}(\Omega), \frac{6}{5}<p<\infty$ and $\boldsymbol{f}(\boldsymbol{h})=\boldsymbol{h}\left(\int_{\Omega}|\boldsymbol{h}|^{2}\right)^{\frac{\sigma-2}{2}}$, with $\sigma \geq 1$, problem (2) has a solution $\boldsymbol{h} \in L^{p}\left(0, T ; \mathbb{W}^{p}(\Omega)\right) \cap \mathscr{C}([0, T] ; \boldsymbol{H}(\operatorname{div} 0, \Omega)) \cap H^{1}\left(0, T ; \boldsymbol{L}^{2}(\Omega)\right)$ for any positive $T$, if $\sigma \leq 2$ or $2<\sigma<p$, and for a small enough $T>0$, otherwise.

Proof. By Propositions 8 and 9 we conclude that the function $\tilde{S}$ is continuous and compact.

We start by analyzing the existence of global solution, applying the Leray-Schauder Theorem, proving that the set

$$
\{\boldsymbol{\varphi} \in \mathscr{C}([0, T] ; \boldsymbol{H}(\operatorname{div} 0, \Omega)): \varphi=\lambda \tilde{S}(\varphi) \text { for some } \lambda \in(0,1]\}
$$

is bounded independently of $\lambda$.

Let $\boldsymbol{\varphi}=\lambda \boldsymbol{h}_{\boldsymbol{\varphi}}$. We consider two different cases. 
- The case $1 \leq \sigma<2$

Using (8), we get

$$
\int_{\Omega}\left|\boldsymbol{h}_{\varphi}(t)\right|^{2} \leq 2 \lambda^{\sigma-1} \int_{0}^{t}\left(\int_{\Omega}\left|\boldsymbol{h}_{\varphi}\right|^{2}\right)^{\frac{\sigma}{2}}+\int_{\Omega}\left|\boldsymbol{h}_{0}\right|^{2} .
$$

So

$$
\begin{aligned}
\int_{\Omega}|\boldsymbol{\varphi}(t)|^{2}=\lambda^{2} \int_{\Omega}\left|\boldsymbol{h}_{\boldsymbol{\varphi}}(t)\right|^{2} \leq 2 \lambda^{\sigma+1} \int_{0}^{t}\left(\int_{\Omega}\left|\frac{\boldsymbol{\varphi}}{\lambda}\right|^{2}\right)^{\frac{\sigma}{2}}+\lambda^{2} \int_{\Omega}\left|\boldsymbol{h}_{0}\right|^{2} \\
\\
\leq 2 \lambda T\|\boldsymbol{\varphi}\|_{\mathscr{C}\left([0, T] ; \boldsymbol{L}^{2}(\Omega)\right)}^{\sigma}+\lambda^{2}\left\|\boldsymbol{h}_{0}\right\|_{\boldsymbol{L}^{2}(\Omega)}^{2} .
\end{aligned}
$$

Hence

$$
\|\boldsymbol{\varphi}\|_{\mathscr{C}\left([0, T] ; \boldsymbol{L}^{2}(\Omega)\right)}^{2} \leq 2 T\|\boldsymbol{\varphi}\|_{\mathscr{C}\left([0, T] ; \boldsymbol{L}^{2}(\Omega)\right)}^{\sigma}+\left\|\boldsymbol{h}_{0}\right\|_{\boldsymbol{L}^{2}(\Omega)}^{2},
$$

concluding that

$$
\|\boldsymbol{\varphi}\|_{\mathscr{C}\left([0, T] ; \boldsymbol{L}^{2}(\Omega)\right)} \leq \max \left\{1,\left(2 T+\left\|\boldsymbol{h}_{0}\right\|_{\boldsymbol{L}^{2}(\Omega)}^{2}\right)^{\frac{1}{2-\sigma}}\right\}
$$

- The case $2 \leq \sigma<p$

By inequality (3) and since $\sigma<p$, we have

$$
\left(\int_{\Omega}\left|\boldsymbol{h}_{\varphi}(t)\right|^{2}\right)^{\frac{\sigma}{2}} \leq C\left(\int_{\Omega}\left|\nabla \times \boldsymbol{h}_{\varphi}(t)\right|^{p}\right)^{\frac{\sigma}{p}} \leq \frac{1}{2} \int_{\Omega}\left|\nabla \times \boldsymbol{h}_{\varphi}(t)\right|^{p}+C_{1}
$$

and by inequality (9),

$$
\frac{1}{2} \int_{\Omega}\left|\boldsymbol{h}_{\boldsymbol{\varphi}}(t)\right|^{2}+\int_{Q_{t}}\left|\nabla \times \boldsymbol{h}_{\boldsymbol{\varphi}}\right|^{p} \leq \frac{1}{2} \int_{\Omega}\left|\boldsymbol{h}_{0}\right|^{2}+\frac{\lambda^{\sigma-1}}{2} \int_{0}^{t}\left(\int_{\Omega}\left|\nabla \times \boldsymbol{h}_{\boldsymbol{\varphi}}(t)\right|^{p}+2 C_{1}\right),
$$

SO

$$
\|\boldsymbol{\varphi}\|_{\mathscr{C}\left([0, T] ; \boldsymbol{L}^{2}(\Omega)\right)}=\lambda\left\|\boldsymbol{h}_{\boldsymbol{\varphi}}\right\|_{\mathscr{C}\left([0, T] ; \boldsymbol{L}^{2}(\Omega)\right)} \leq\left(\left\|\boldsymbol{h}_{0}\right\|_{\boldsymbol{L}^{2}(\Omega)}^{2}+2 C_{1}\right)^{\frac{1}{2}} .
$$

We proceed with the proof of existence of local solution when $\sigma \geq \max \{2, p\}$, using the Schauder Fixed Point Theorem. From inequality (9) we obtain

$$
\begin{aligned}
\frac{1}{2} \int_{\Omega}\left|\boldsymbol{h}_{\boldsymbol{\varphi}}(t)\right|^{2} & \leq \frac{1}{2} \int_{\Omega}\left|\boldsymbol{h}_{0}\right|^{2}+C \int_{0}^{t}\|\boldsymbol{\varphi}(\tau)\|_{\mathscr{C}\left([0, T] ; \boldsymbol{L}^{2}(\Omega)\right)}^{2(\sigma-1)}+\frac{1}{4 T} \int_{0}^{t}\left\|\boldsymbol{h}_{\boldsymbol{\varphi}}(\tau)\right\|_{\boldsymbol{L}^{2}(\Omega)}^{2} \\
& \leq C T\|\boldsymbol{\varphi}\|_{\mathscr{C}\left([0, T] ; \boldsymbol{L}^{2}(\Omega)\right)}^{2(\sigma-1)}+\frac{1}{4}\left\|\boldsymbol{h}_{\boldsymbol{\varphi}}\right\|_{L^{\infty}\left(0, T ; \boldsymbol{L}^{2}(\Omega)\right)}^{2}+\frac{1}{2} \int_{\Omega}\left|\boldsymbol{h}_{0}\right|^{2}
\end{aligned}
$$

and, finally,

$$
\left\|\boldsymbol{h}_{\boldsymbol{\varphi}}\right\|_{L^{\infty}\left(0, T ; \boldsymbol{L}^{2}(\Omega)\right)}^{2} \leq 2 \int_{\Omega}\left|\boldsymbol{h}_{0}\right|^{2}+4 C T\|\boldsymbol{\varphi}\|_{\mathscr{C}\left([0, T] ; \boldsymbol{L}^{2}(\Omega)\right)}^{2(\sigma-1)} .
$$

The last inequality proves that for positive big enough $R$, there exists a sufficiently small $T$ such that $\tilde{S}\left(D_{R}(0)\right) \subseteq D_{R}(0)$. Any fixed point of $\tilde{S}$ solves problem (2). The additional regularity of the solution is a consequence of the estimates obtained before.

Remark 11. For $p>3$ any solution $\boldsymbol{h}$ of problem (1) belongs to $\mathscr{C}\left(\bar{Q}_{T}\right)$. This is an immediate consequence of Theorem 5 of [10], choosing $X=\mathbb{W}^{p}(\Omega), B=\mathscr{C}(\bar{\Omega})$ and $Y=\boldsymbol{L}^{2}(\Omega)$.

For $p=3, \boldsymbol{h} \in \boldsymbol{L}^{\infty}\left(Q_{T}\right)$ since $\mathbb{W}^{3}(\Omega) \subseteq \boldsymbol{L}^{\infty}(\Omega)$. 


\subsection{Blow-up}

In this section, we study sufficient conditions for the blow-up of solutions $\boldsymbol{h}$ of problem (2). Let us introduce the energy function

$$
E(t)=\frac{1}{p}\|\nabla \times \boldsymbol{h}(t)\|_{\boldsymbol{L}^{p}(\Omega)}^{p}-\frac{1}{\sigma}\|\boldsymbol{h}(t)\|_{\boldsymbol{L}^{2}(\Omega)}^{\sigma} .
$$

Theorem 12. Let $\boldsymbol{h}_{0} \in \boldsymbol{L}^{2}(\Omega), \frac{6}{5}<p<\infty$. Assume that

$$
E(0)=\frac{1}{p}\left\|\nabla \times \boldsymbol{h}_{0}\right\|_{\boldsymbol{L}^{p}(\Omega)}^{p}-\frac{1}{\sigma}\left\|\boldsymbol{h}_{0}\right\|_{\boldsymbol{L}^{2}(\Omega)}^{\sigma} \leq 0
$$

and

$$
\max \{2, p\}<\sigma .
$$

Then the solution $\boldsymbol{h}$ of problem (1) blows up (in the sense that $\|\boldsymbol{h}(t)\|_{\boldsymbol{L}^{2}(\Omega)}$ becomes unbounded) on the finite interval $\left(0, t_{\max }\right)$, with

$$
t_{\max }=\frac{\lambda \sigma}{(\sigma-2)(\lambda \sigma-1)\left\|\boldsymbol{h}_{0}\right\|_{\boldsymbol{L}^{2}(\Omega)}^{\sigma-2}} .
$$

Proof. Using, as before, $\partial_{t} \boldsymbol{h}$ as a test function in equation (5a), we obtain

$$
\int_{\Omega}\left|\partial_{t} \boldsymbol{h}(t)\right|^{2}+E^{\prime}(t)=0
$$

Integrating the last equation and taking into account(16), we obtain

$$
E(t)+\int_{Q_{t}}\left|\partial_{t} \boldsymbol{h}\right|^{2}=E(0) \leq 0 .
$$

Using $\boldsymbol{h}$ as a test function in (2a) and integrating in time, we obtain

$$
\frac{1}{2} \int_{\Omega}|\boldsymbol{h}(t)|^{2}+\int_{Q_{t}}|\nabla \times \boldsymbol{h}|^{p}=\frac{1}{2} \int_{\Omega}\left|\boldsymbol{h}_{0}\right|^{2}+\int_{0}^{t}\left(\int_{\Omega}|\boldsymbol{h}|^{2}\right)^{\frac{\sigma}{2}} .
$$

Denoting $F(t)=\frac{1}{2} \int_{Q_{t}}|\boldsymbol{h}|^{2}$ we have $F^{\prime}(t)=\frac{1}{2} \int_{\Omega}|\boldsymbol{h}(t)|^{2}$ and so

$$
F^{\prime}(t)=-\int_{Q_{t}}|\nabla \times \boldsymbol{h}|^{p}+\frac{1}{2} \int_{\Omega}\left|\boldsymbol{h}_{0}\right|^{2}+\int_{0}^{t}\left(\int_{\Omega}|\boldsymbol{h}|^{2}\right)^{\frac{\sigma}{2}} .
$$

Thus

$$
F^{\prime \prime}(t)=\int_{\Omega} \partial_{t} \boldsymbol{h}(t) \cdot \boldsymbol{h}(t)=-\int_{\Omega}|\nabla \times \boldsymbol{h}(t)|^{p}+\left(\int_{\Omega}|\boldsymbol{h}(t)|^{2}\right)^{\frac{\sigma}{2}} .
$$

For $\lambda \in \mathbb{R}^{+}$, as, by (17), $E(t) \leq 0$, we have

$$
\begin{aligned}
\lambda F^{\prime \prime}(t) \geq E(t)+\lambda\left(\left(\int_{\Omega}|\boldsymbol{h}(t)|^{2}\right)^{\frac{\sigma}{2}}-\int_{\Omega}|\nabla \times \boldsymbol{h}(t)|^{p}\right) & \\
& =\left(\frac{1}{p}-\lambda\right) \int_{\Omega}|\nabla \times \boldsymbol{h}(t)|^{p}+\left(\lambda-\frac{1}{\sigma}\right)\left(\int_{\Omega}|\boldsymbol{h}(t)|^{2}\right)^{\frac{\sigma}{2}} .
\end{aligned}
$$

Choosing $\lambda$ such that $\frac{1}{\sigma}<\lambda<\frac{1}{p}$, we obtain

$$
\lambda F^{\prime \prime} \geq\left(\lambda-\frac{1}{\sigma}\right) 2^{\frac{\sigma}{2}}\left(F^{\prime}\right)^{\frac{\sigma}{2}}
$$


and so

$$
F^{\prime \prime} \geq C\left(F^{\prime}\right)^{\frac{\sigma}{2}}, \quad C=\frac{1}{\lambda}\left(\lambda-\frac{1}{\sigma}\right) 2^{\frac{\sigma}{2}} .
$$

Integrating the last ordinary differential inequality, as $\sigma>2$, we arrive at

$$
\frac{\left(F^{\prime}(0)\right)^{\frac{\sigma-2}{2}}}{1-\frac{\sigma-2}{2} C t\left(F^{\prime}(0)\right)^{\frac{\sigma-2}{2}}} \leq\left(F^{\prime}(t)\right)^{\frac{\sigma-2}{2}}
$$

or

$$
\frac{\left\|\boldsymbol{h}_{0}\right\|^{2}}{\left(1-\frac{\sigma-2}{2} C t 2^{-\frac{\sigma-2}{2}}\left\|\boldsymbol{h}_{0}\right\|_{\boldsymbol{L}^{2}(\Omega)}^{\sigma-2}\right)^{\frac{2}{\sigma-2}}} \leq\|\boldsymbol{h}(t)\|_{\boldsymbol{L}^{2}(\Omega)}^{2} .
$$

Hence

$$
t_{\max }=\frac{\lambda \sigma}{(\sigma-2)(\lambda \sigma-1)\left\|\boldsymbol{h}_{0}\right\|_{\boldsymbol{L}^{2}(\Omega)}^{\sigma-2}} .
$$

\section{Finite time extinction: $\boldsymbol{f}(\boldsymbol{h})=-\boldsymbol{h}\left(\int_{\Omega}|\boldsymbol{h}|^{k}\right)^{-\lambda}$}

In this section, we assume that $\frac{6}{5}<p<\infty$ and we find sufficient conditions on $k, \lambda \in \mathbb{R}^{+}$for the existence of solution of problem (2). The proof of existence will be done using the Galerkin method. We also study sufficient conditions for the finite time extinction of solutions.

We start by setting a topological basis $\left(\psi_{n}\right)_{n}$ of $\mathbb{W}^{p}(\Omega)$ such that $\boldsymbol{\psi}_{n} \in \boldsymbol{L}^{\infty}(\Omega)$, for all $n \in \mathbb{N}$. Assuming that $\boldsymbol{h}_{0} \in \mathbb{W}^{p}(\Omega)$, let $\boldsymbol{h}_{m, 0}$ be an approximation, belonging to $\left\langle\boldsymbol{\psi}_{1}, \ldots, \boldsymbol{\psi}_{m}\right\rangle$, of $\boldsymbol{h}_{0}$, in $\mathbb{W}^{p}(\Omega)$. Denoting

$$
\boldsymbol{h}_{m}(t)=\sum_{i=1}^{m} \lambda_{i}^{m}(t) \boldsymbol{\psi}_{i}
$$

the system of ODE's in the unknowns $\lambda_{1}^{m}, \ldots, \lambda_{m}^{m}$, for $t \in(0, T)$,

$$
\begin{aligned}
& \int_{\Omega} \partial_{t} \boldsymbol{h}_{m}(t) \cdot \boldsymbol{\psi}_{i}+\int_{\Omega}\left|\nabla \times \boldsymbol{h}_{m}(t)\right|^{p-2} \nabla \times \boldsymbol{h}_{m}(t) \cdot \nabla \times \boldsymbol{\psi}_{i} \\
&+\left(\int_{\Omega}\left|\boldsymbol{h}_{m}(t)\right|^{k}\right)^{-\lambda} \int_{\Omega} \boldsymbol{h}_{m}(t) \cdot \boldsymbol{\psi}_{i}=0, \quad i=1, \ldots, m, \\
& \boldsymbol{h}_{m}(\cdot, 0)=\boldsymbol{h}_{m, 0} \quad \text { in } \Omega,
\end{aligned}
$$

has a unique solution $\boldsymbol{h}_{m} \in \mathscr{C}^{\infty}\left(0, T ;\left\langle\boldsymbol{\psi}_{1}, \ldots, \boldsymbol{\psi}_{m}\right\rangle\right)$.

The above system is equivalent to the following one:

$$
\begin{aligned}
& \int_{\Omega} \partial_{t} \boldsymbol{h}_{m}(t) \cdot \boldsymbol{\psi}(t)+\int_{\Omega}\left|\nabla \times \boldsymbol{h}_{m}(t)\right|^{p-2} \nabla \times \boldsymbol{h}_{m}(t) \cdot \nabla \times \boldsymbol{\psi}(t) \\
& \quad+\left(\int_{\Omega}\left|\boldsymbol{h}_{m}(t)\right|^{k}\right)^{-\lambda} \int_{\Omega} \boldsymbol{h}_{m}(t) \cdot \boldsymbol{\psi}(t)=0, \forall \boldsymbol{\psi} \in \mathscr{C}^{\infty}\left(0, T ;\left\langle\boldsymbol{\psi}_{1}, \ldots, \boldsymbol{\psi}_{m}\right\rangle\right), \\
& \boldsymbol{h}_{m}(\cdot, 0)=\boldsymbol{h}_{m, 0} \quad \text { in } \Omega .
\end{aligned}
$$

Using $\boldsymbol{h}_{m}$ as a test function in (18) we obtain

$$
\frac{1}{2} \int_{\Omega}\left|\boldsymbol{h}_{m}(t)\right|^{2}+\int_{Q_{t}}\left|\nabla \times \boldsymbol{h}_{m}\right|^{p}+\int_{0}^{t}\left(\int_{\Omega}\left|\boldsymbol{h}_{m}\right|^{k}\right)^{-\lambda}\left(\int_{\Omega}\left|\boldsymbol{h}_{m}\right|^{2}\right)=\frac{1}{2} \int_{\Omega}\left|\boldsymbol{h}_{0}\right|^{2},
$$

from which we derive the following a priori estimates, for a positive constant $C$ independent of $m$,

$$
\left\|\boldsymbol{h}_{m}\right\|_{L^{\infty}\left(0, T ; \boldsymbol{L}^{2}(\Omega)\right)} \leq C, \quad\left\|\boldsymbol{h}_{m}\right\|_{L^{p}\left(0, T ; \mathbb{W}^{p}(\Omega)\right)} \leq C .
$$




\subsection{Existence}

The proof of existence of solution will be split in three different cases, according to different values of $k$. For $k=2$ uniqueness is proved.

\subsubsection{The case $k=2$}

Theorem 13. Assume that $\boldsymbol{h}_{0} \in \mathbb{W}^{p}(\Omega), \frac{6}{5}<p<\infty$ and $0<\lambda<1$. Then problem (2) has a solution

$$
\boldsymbol{h} \in L^{\infty}\left(0, T ; \boldsymbol{L}^{2}(\Omega)\right) \cap L^{\infty}\left(0, T ; \mathbb{W}^{p}(\Omega)\right) \cap H^{1}\left(0, T ; \boldsymbol{L}^{2}(\Omega)\right)
$$

The solution is unique if $\lambda \leq \frac{1}{2}$.

Proof. Let $\boldsymbol{h}_{m}$ be the solution of problem (18) and using $\partial_{t} \boldsymbol{h}_{m}$ as a test function in (18), we obtain, for $\lambda<1$,

$$
\begin{aligned}
\int_{Q_{t}}\left|\partial_{t} \boldsymbol{h}_{m}\right|^{2}+\frac{1}{p} \int_{\Omega}\left|\nabla \times \boldsymbol{h}_{m}(t)\right|^{p}+\frac{1}{2(1-\lambda)} & \left(\int_{\Omega}\left|\boldsymbol{h}_{m}(t)\right|^{2}\right)^{1-\lambda} \\
& =\frac{1}{p} \int_{\Omega}\left|\nabla \times \boldsymbol{h}_{m, 0}\right|^{p}+\frac{1}{2(1-\lambda)}\left(\int_{\Omega}\left|\boldsymbol{h}_{m, 0}\right|^{2}\right)^{1-\lambda} .
\end{aligned}
$$

From (19) and (20) we conclude that there exists a function $\boldsymbol{h}$ belonging to $L^{\infty}\left(0, T ; \boldsymbol{L}^{2}(\Omega)\right) \cap$ $L^{\infty}\left(0, T ; \mathbb{W}^{p}(\Omega)\right) \cap H^{1}\left(0, T ; \boldsymbol{L}^{2}(\Omega)\right)$ such that, at least for a subsequence,

$$
\begin{aligned}
& \boldsymbol{h}_{m} \longrightarrow \boldsymbol{h} \quad \text { in } L^{\infty}\left(0, T ; \boldsymbol{L}^{2}(\Omega)\right) \text { - weak*, } \\
& \nabla \times \boldsymbol{h}_{m} \longrightarrow \nabla \times \boldsymbol{h} \quad \text { in } L^{\infty}\left(0, T ; \boldsymbol{L}^{p}(\Omega)\right) \text { - weak*, } \\
& \left|\nabla \times \boldsymbol{h}_{m}\right|^{p-2} \nabla \times \boldsymbol{h}_{m} \longrightarrow \chi \quad \text { in } L^{\infty}\left(0, T ; \boldsymbol{L}^{p^{\prime}}(\Omega)\right) \text { - weak*, } \\
& \left.\partial_{t} \boldsymbol{h}_{m} \longrightarrow \partial_{t} \boldsymbol{h} \quad \text { in } \boldsymbol{L}^{2}(Q)\right) \text { - weak. }
\end{aligned}
$$

Observe that, given $\delta>0$, for $t \in(0, T-\delta)$ we have

$$
\int_{\Omega}\left|\boldsymbol{h}_{m}(t+\delta)-\boldsymbol{h}_{m}(t)\right|^{2}=\int_{\Omega}\left|\int_{t}^{t+\delta} \partial_{t} \boldsymbol{h}_{m}(\tau)\right|^{2} \leq \delta\left\|\partial_{t} \boldsymbol{h}_{m}\right\|_{\boldsymbol{L}^{2}(Q)}^{2} \leq \delta C .
$$

Denoting $\boldsymbol{X}=\mathbb{W}^{p}(\Omega)$ and $\boldsymbol{B}=\boldsymbol{Y}=\boldsymbol{H}(\operatorname{div} 0, \Omega)$ we observe that $\left\{h_{m}: m \in \mathbb{N}\right\}$ is bounded in

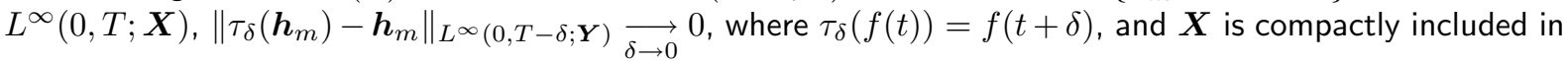
$\boldsymbol{B}$, as $p>\frac{6}{5}$. Applying, as before, Theorem 5 [10], we conclude that $\left\{h_{m}: m \in \mathbb{N}\right\}$ is contained in a compact subset of $\mathscr{C}([0, T] ; \boldsymbol{B})$. So, at least for a subsequence,

$$
\int_{\Omega}\left|\boldsymbol{h}_{m}(t)\right|^{2} \longrightarrow \int_{\Omega}|\boldsymbol{h}(t)|^{2}, \quad \text { for all } t \in[0, T] .
$$

Integrating (18) in time and passing to the limit in $m$, for test functions $\boldsymbol{\psi} \in L^{p}\left(0, T ;\left\langle\boldsymbol{\psi}_{1}, \ldots, \boldsymbol{\psi}_{k}\right\rangle\right)$, $k \in \mathbb{N}$ fixed, we obtain

$$
\begin{aligned}
& \int_{Q_{T}} \partial_{t} \boldsymbol{h} \cdot \boldsymbol{\psi}+\int_{Q_{T}} \boldsymbol{\chi} \cdot \nabla \times \boldsymbol{\psi}+\int_{0}^{T}\left(\int_{\Omega}|\boldsymbol{h}|^{2}\right)^{-\lambda}\left(\int_{\Omega} \boldsymbol{h} \cdot \boldsymbol{\psi}\right)=0, \\
& \boldsymbol{h}(\cdot, 0)=\boldsymbol{h}_{0} \quad \text { in } \Omega
\end{aligned}
$$

and, afterwards, by density, for all $\boldsymbol{\psi} \in L^{p}\left(0, t ; \mathbb{W}^{p}(\Omega)\right)$.

The identification of $\boldsymbol{\chi}$ with $|\nabla \times \boldsymbol{h}|^{p-2} \nabla \times \boldsymbol{h}$ follows the arguments of Lions (see [11], p 160) for bounded, hemicontinuous, coercive and monotone operators. Finally, standard arguments allow us to rewrite (21a) as the weak formulation introduced in (2) for $f(\boldsymbol{h})=-\boldsymbol{h}\left(\int_{\Omega}|\boldsymbol{h}|^{2}\right)^{-\lambda}$. 
To prove the uniqueness of solution in the case $\lambda \leq \frac{1}{2}$, let $\boldsymbol{h}_{1}$ and $\boldsymbol{h}_{2}$ be two solutions of (2). Use $\boldsymbol{h}_{1}-\boldsymbol{h}_{2}$ as a test function in the problem solved by $\boldsymbol{h}_{1}$ and by $\boldsymbol{h}_{2}$. Then we get, after subtraction, for $C_{p}$ defined in (13), in the case $p \geq 2$,

$$
\begin{aligned}
\frac{1}{2} \int_{\Omega} \mid \boldsymbol{h}_{1}(t) & -\left.\boldsymbol{h}_{2}(t)\right|^{2}+C_{p} \int_{Q_{t}}\left|\nabla \times\left(\boldsymbol{h}_{1}-\boldsymbol{h}_{2}\right)\right|^{p} \\
& +\int_{0}^{t}\left(\int_{\Omega}\left|\boldsymbol{h}_{1}\right|^{2}\right)^{-\lambda}\left(\int_{\Omega} \boldsymbol{h}_{1} \cdot\left(\boldsymbol{h}_{1}-\boldsymbol{h}_{2}\right)\right)-\int_{0}^{t}\left(\int_{\Omega}\left|\boldsymbol{h}_{2}\right|^{2}\right)^{-\lambda}\left(\int_{\Omega} \boldsymbol{h}_{2} \cdot\left(\boldsymbol{h}_{1}-\boldsymbol{h}_{2}\right)\right) \leq 0
\end{aligned}
$$

and, for $C_{p}$ defined in (14), in the case $\frac{6}{5}<p<2$,

$$
\begin{aligned}
\frac{1}{2} \int_{\Omega} \mid \boldsymbol{h}_{1}(t) & -\left.\boldsymbol{h}_{2}(t)\right|^{2}+C_{p} \int_{Q_{t}}\left(\left|\nabla \times \boldsymbol{h}_{1}\right|+\left|\nabla \times \boldsymbol{h}_{2}\right|\right)^{p-2}\left|\nabla \times\left(\boldsymbol{h}_{1}-\boldsymbol{h}_{2}\right)\right|^{2} \\
& +\int_{0}^{t}\left(\int_{\Omega}\left|\boldsymbol{h}_{1}\right|^{2}\right)^{-\lambda}\left(\int_{\Omega} \boldsymbol{h}_{1} \cdot\left(\boldsymbol{h}_{1}-\boldsymbol{h}_{2}\right)\right)-\int_{0}^{t}\left(\int_{\Omega}\left|\boldsymbol{h}_{2}\right|^{2}\right)^{-\lambda}\left(\int_{\Omega} \boldsymbol{h}_{2} \cdot\left(\boldsymbol{h}_{1}-\boldsymbol{h}_{2}\right)\right) \leq 0 .
\end{aligned}
$$

Calling $y_{1}(t)=\int_{\Omega}\left|\boldsymbol{h}_{1}(t)\right|^{2}$ and $y_{2}(t)=\int_{\Omega}\left|\boldsymbol{h}_{2}(t)\right|^{2}$, we obtain, for $\lambda \leq \frac{1}{2}$,

$$
\begin{aligned}
0 & \geq \int_{0}^{t}\left(\int_{\Omega}\left|\boldsymbol{h}_{1}\right|^{2}\right)^{-\lambda}\left(\int_{\Omega} \boldsymbol{h}_{1} \cdot\left(\boldsymbol{h}_{1}-\boldsymbol{h}_{2}\right)\right)-\int_{0}^{t}\left(\int_{\Omega}\left|\boldsymbol{h}_{2}\right|^{2}\right)^{-\lambda}\left(\int_{\Omega} \boldsymbol{h}_{2} \cdot\left(\boldsymbol{h}_{1}-\boldsymbol{h}_{2}\right)\right) \\
& \geq \int_{0}^{t}\left(y_{1}^{1-\lambda}+y_{2}^{1-\lambda}-y_{1}^{\frac{1}{2}-\lambda} y_{2}^{\frac{1}{2}}-y_{2}^{\frac{1}{2}-\lambda} y_{1}^{\frac{1}{2}}\right) \\
& =\int_{0}^{t}\left(y_{1}^{\frac{1}{2}-\lambda}-y_{2}^{\frac{1}{2}-\lambda}\right)\left(y_{1}^{\frac{1}{2}}-y_{2}^{\frac{1}{2}}\right) \geq 0
\end{aligned}
$$

From the above inequality we conclude that $y_{1}(t)=y_{2}(t)=0$ for a.e. $t \in(0, T)$ and

$$
\int_{0}^{t}\left(\int_{\Omega}\left|\boldsymbol{h}_{1}\right|^{2}\right)^{-\lambda}\left(\int_{\Omega} \boldsymbol{h}_{1} \cdot\left(\boldsymbol{h}_{1}-\boldsymbol{h}_{2}\right)\right)-\int_{0}^{t}\left(\int_{\Omega}\left|\boldsymbol{h}_{2}\right|^{2}\right)^{-\lambda}\left(\int_{\Omega} \boldsymbol{h}_{2} \cdot\left(\boldsymbol{h}_{1}-\boldsymbol{h}_{2}\right)\right)=0 .
$$

Consequently,

$$
\frac{1}{2} \int_{\Omega}\left|\boldsymbol{h}_{1}(t)-\boldsymbol{h}_{2}(t)\right|^{2} \leq 0
$$

which implies that $\boldsymbol{h}_{1}=\boldsymbol{h}_{2}$ a.e. in $Q$.

\subsubsection{The case $k>2$}

Theorem 14. Suppose that $\boldsymbol{h}_{0} \in \mathbb{W}^{p}(\Omega), \frac{6}{5}<p<\infty, 0<\lambda<\frac{1}{k}$, where $2<k<\frac{3 p}{3-p}$ if $p<3$ and $2<k$ if $p \geq 3$.

Then problem (2) has a solution

$$
\boldsymbol{h} \in L^{\infty}\left(0, T ; \boldsymbol{L}^{2}(\Omega)\right) \cap L^{\infty}\left(0, T ; \mathbb{W}^{p}(\Omega)\right) \cap H^{1}\left(0, T ; \boldsymbol{L}^{2}(\Omega)\right) .
$$

Proof. Let $\boldsymbol{h}_{m}$ be the solution of problem (18) and using $\partial_{t} \boldsymbol{h}_{m}$ as a test function in (18), we obtain

$$
\begin{aligned}
\int_{Q_{t}}\left|\partial_{t} \boldsymbol{h}_{m}\right|^{2}+\frac{1}{p} \int_{\Omega}\left|\nabla \times \boldsymbol{h}_{m}(t)\right|^{p} & =\frac{1}{p} \int_{\Omega}\left|\nabla \times \boldsymbol{h}_{m, 0}\right|^{p}-\int_{0}^{t}\left(\int_{\Omega}\left|\boldsymbol{h}_{m}\right|^{k}\right)^{-\lambda}\left(\int_{\Omega} \boldsymbol{h}_{m} \cdot \partial_{t} \boldsymbol{h}_{m}\right) \\
& \leq \frac{1}{p} \int_{\Omega}\left|\nabla \times \boldsymbol{h}_{m, 0}\right|^{p}+|\Omega|^{\frac{\lambda(k-2)}{2}} \int_{0}^{t}\left(\int_{\Omega}\left|\boldsymbol{h}_{m}\right|^{2}\right)^{\frac{1-k \lambda}{2}}\left(\int_{\Omega}\left|\partial_{t} \boldsymbol{h}_{m}\right|^{2}\right)^{\frac{1}{2}} \\
& \leq \frac{1}{p} \int_{\Omega}\left|\nabla \times \boldsymbol{h}_{m, 0}\right|^{p}+C(|\Omega|) T\left\|\boldsymbol{h}_{m}\right\|_{L^{\infty}\left(0, T ; L^{2}(\Omega)\right)}^{1-\lambda k}+\frac{1}{2} \int_{Q_{t}}\left|\partial_{t} \boldsymbol{h}_{m}\right|^{2} .
\end{aligned}
$$


As, by (19), $\left\|\boldsymbol{h}_{m}\right\|_{L^{\infty}\left(0, T ; \boldsymbol{L}^{2}(\Omega)\right)}$ is bounded independently of $m$, there exists a positive constant $C$ such that

$$
\left\|\partial_{t} \boldsymbol{h}_{m}\right\|_{\boldsymbol{L}^{2}(Q)} \leq C, \quad\left\|\nabla \times \boldsymbol{h}_{m}\right\|_{L^{\infty}\left(0, T ; \boldsymbol{L}^{p}(\Omega)\right)} \leq C .
$$

As in the case $k=2$, the set $\left\{\boldsymbol{h}_{m}: m \in \mathbb{N}\right\}$ is contained in a compact subset of $\mathscr{C}([0, T) ; \boldsymbol{H}(\operatorname{div} 0, \Omega))$ and this implies that

$$
\int_{\Omega}\left|\boldsymbol{h}_{m}(t)\right|^{2} \longrightarrow \int_{\Omega}|\boldsymbol{h}(t)|^{2} .
$$

But, as $k$ is less than or equal to the critical exponent $q$ defined in Lemma 2, there exist positive constants $C_{1}$ and $C_{2}$ such that

$$
\left\|\boldsymbol{h}_{m}(t)\right\|_{\boldsymbol{L}^{k}(\Omega)} \leq C_{1}\left\|\nabla \times \boldsymbol{h}_{m}\right\|_{L^{\infty}\left(0, T ; \boldsymbol{L}^{p}(\Omega)\right)} \leq C_{2},
$$

and Lebesgue Theorem implies that

$$
\int_{\Omega}\left|\boldsymbol{h}_{m}(t)\right|^{k} \longrightarrow \int_{\Omega}|\boldsymbol{h}(t)|^{k}
$$

The conclusion follows as in the proof of Theorem 13.

\subsubsection{The case $1 \leq k<2$}

Theorem 15. Suppose that $\boldsymbol{h}_{0} \in \mathbb{W}^{p}(\Omega), \frac{6}{5}<p<\infty$ and $k, \lambda$ are such that for

$$
p<3, \theta=\frac{\frac{1}{k}-\frac{1}{2}}{\frac{1}{k}-\frac{3-p}{3 p}} \text { and } 0<\lambda<\frac{1-\theta}{k} ; \quad p \geq 3, \theta \in(0,1) \text { and } 0<\lambda<\frac{1}{k} .
$$

Then problem (2) has a solution

$$
\boldsymbol{h} \in L^{\infty}\left(0, T ; \boldsymbol{L}^{2}(\Omega)\right) \cap L^{\infty}\left(0, T ; \mathbb{W}^{p}(\Omega)\right) \cap H^{1}\left(0, T ; \boldsymbol{L}^{2}(\Omega)\right) .
$$

Proof. Using $\partial_{t} \boldsymbol{h}_{m}(t)$ as a test function in problem (18) we have, as before,

$$
\int_{Q_{t}}\left|\partial_{t} \boldsymbol{h}_{m}\right|^{2}+\frac{1}{p} \int_{\Omega}\left|\nabla \times \boldsymbol{h}_{m}(t)\right|^{p}=\frac{1}{p} \int_{\Omega}\left|\nabla \times \boldsymbol{h}_{m, 0}\right|^{p}-\int_{0}^{t}\left(\int_{\Omega}\left|\boldsymbol{h}_{m}\right|^{k}\right)^{-\lambda}\left(\int_{\Omega} \boldsymbol{h}_{m} \cdot \partial_{t} \boldsymbol{h}_{m}\right) .
$$

We consider first the case $p<3$. Using Lemma 3, Hölder and Young inequalities we obtain,

$$
\begin{aligned}
\mid\left(\int_{\Omega}\left|\boldsymbol{h}_{m}(t)\right|^{k}\right)^{-\lambda}\left(\int_{\Omega} \boldsymbol{h}_{m}(t)\right. & \left.\cdot \partial_{t} \boldsymbol{h}_{m}(t)\right) \mid \\
& \leq\left(\int_{\Omega}\left|\boldsymbol{h}_{m}(t)\right|^{k}\right)^{-\lambda}\left(\int_{\Omega}\left|\boldsymbol{h}_{m}(t)\right|\right)^{\frac{1}{2}}\left(\int_{\Omega}\left|\partial_{t} \boldsymbol{h}_{m}(t)\right|^{2}\right)^{\frac{1}{2}} \\
& \leq C_{1}\left(\int_{\Omega}\left|\boldsymbol{h}_{m}(t)\right|^{k}\right)^{-2 \lambda} \int_{\Omega}\left|\boldsymbol{h}_{m}(t)\right|^{2}+\frac{1}{2} \int_{\Omega}\left|\partial_{t} \boldsymbol{h}_{m}(t)\right|^{2} \\
& \leq C_{1}\left(\int_{\Omega}\left|\boldsymbol{h}_{m}(t)\right|^{k}\right)^{2\left(\frac{1-\theta}{k}-\lambda\right)}\left(\int_{\Omega}\left|\nabla \times \boldsymbol{h}_{m}(t)\right|^{p}\right)^{\frac{2 \theta}{p}}+\frac{1}{2} \int_{\Omega}\left|\partial_{t} \boldsymbol{h}_{m}(t)\right|^{2} .
\end{aligned}
$$

Noticing that $p>2 \theta$ because $\frac{6}{5} \leq p<3$ and $1 \leq k<2$, we obtain

$$
\left(\int_{\Omega}\left|\boldsymbol{h}_{m}(t)\right|^{k}\right)^{2\left(\frac{1-\theta}{k}-\lambda\right)}\left(\int_{\Omega}\left|\nabla \times \boldsymbol{h}_{m}(t)\right|^{p}\right)^{\frac{2 \theta}{p}} \leq C_{2}\left(\int_{\Omega}\left|\boldsymbol{h}_{m}(t)\right|^{2}\right)^{\frac{p}{p-2 \theta}\left(\frac{1-\theta}{k}-\lambda\right) k}+\frac{1}{2} \int_{\Omega}\left|\nabla \times \boldsymbol{h}_{m}(t)\right|^{p},
$$

being this inequality true also because, by assumption, $\lambda<\frac{1-\theta}{k}$.

As, by (19), $\left\|\boldsymbol{h}_{m}\right\|_{L^{\infty}\left(0, T ; \boldsymbol{L}^{2}(\Omega)\right)}$ is bounded independently of $m$, by (23), we have

$$
\int_{Q_{t}}\left|\partial_{t} \boldsymbol{h}_{m}\right|^{2}+\frac{1}{p} \int_{\Omega}\left|\nabla \times \boldsymbol{h}_{m}(t)\right|^{p} \leq C+\frac{1}{p} \int_{\Omega}\left|\nabla \times \boldsymbol{h}_{m, 0}\right|^{p}
$$

and the conclusion follows as in the case $k>2$.

The case $p \geq 3$ is simpler and is treated similarly. 


\subsection{Finite time extinction}

We consider two different cases, both with $\lambda>0$ :

i) the case $0<k \leq 2$;

ii) the case $2<k \leq \frac{3 p}{3-p}$ if $p<3$ and $2<k$ if $p \geq 3$.

\subsubsection{The case $0<k \leq 2$}

Theorem 16. Let $\boldsymbol{h}_{0} \in \mathbb{W}^{p}(\Omega), \frac{6}{5}<p<\infty, 0<k \leq 2$ and $\lambda>0$. Define

$$
t_{*}=\frac{|\Omega|^{\frac{\lambda(2-k)}{2}}\left\|\boldsymbol{h}_{0}\right\|_{\boldsymbol{L}^{2}(\Omega)}^{\lambda k}}{\lambda k} .
$$

If $\boldsymbol{h}$ is a solution of problem (2), then, for $t \geq t_{*}$, we have $\|\boldsymbol{h}(t)\|_{\boldsymbol{L}^{2}(\Omega)}=0$.

Proof. We start by noticing that, for $0<k \leq 2$ and $\boldsymbol{f} \in \boldsymbol{L}^{2}(\Omega)$, we have

$$
\int_{\Omega}|\boldsymbol{f}|^{k} \leq|\Omega|^{1-\frac{k}{2}}\left(\int_{\Omega}|\boldsymbol{f}|^{2}\right)^{\frac{k}{2}}
$$

As

$$
\frac{1}{2} \frac{d}{d t} \int_{\Omega}|\boldsymbol{h}(t)|^{2}+\int_{\Omega}|\nabla \times \boldsymbol{h}(t)|^{p}+\left(\int_{\Omega}|\boldsymbol{h}(t)|^{k}\right)^{-\lambda}\left(\int_{\Omega}|\boldsymbol{h}(t)|^{2}\right)=0
$$

we get

$$
\frac{1}{2} \frac{d}{d t} \int_{\Omega}|\boldsymbol{h}(t)|^{2}+|\Omega|^{\frac{\lambda(k-2)}{2}}\left(\int_{\Omega}|\boldsymbol{h}(t)|^{2}\right)^{1-\frac{\lambda k}{2}} \leq 0 .
$$

Calling $y(t)=\int_{\Omega}|\boldsymbol{h}(t)|^{2}, C=2|\Omega|^{\frac{\lambda(k-2)}{2}}$ and $\mu=1-\frac{\lambda k}{2}$, we obtain the differential inequality

$$
y^{\prime}(t)+C y(t)^{\mu} \leq 0
$$

So,

$$
\left(\int_{\Omega}|\boldsymbol{h}(t)|^{2}\right)^{\frac{\lambda k}{2}} \leq-\lambda k|\Omega|^{\frac{\lambda(k-2)}{2}} t+\left(\int_{\Omega}\left|\boldsymbol{h}_{0}\right|^{2}\right)^{\frac{\lambda k}{2}},
$$

concluding that $y(t)=0$ for $t \geq t_{*}$, being $t_{*}$ defined in (24).

\subsubsection{The case $k>2$}

Theorem 17. Let $\boldsymbol{h}_{0} \in \mathbb{W}^{p}(\Omega), \frac{6}{5}<p<\infty, \lambda>0$ and $k$ be such that $2<k \leq \frac{3 p}{3-p}$ if $p<3$ and $2<k$ if $p \geq 3$.

If $\boldsymbol{h}$ is a solution of problem (2), there exists $t_{*}\left(\right.$ see (26)) such that, for $t \geq t_{*}$ we have $\|\boldsymbol{h}(t)\|_{\boldsymbol{L}^{2}(\Omega)}=0$. Proof. Using inequality (4), we have

$$
\left(\int_{\Omega}|\boldsymbol{h}(t)|^{k}\right)^{-\lambda}\left(\int_{\Omega}|\boldsymbol{h}(t)|^{2}\right) \geq C^{-\lambda}\left(\int_{\Omega}|\nabla \times \boldsymbol{h}(t)|^{p}\right)^{-\lambda \frac{k \theta}{p}}\left(\int_{\Omega}|\boldsymbol{h}(t)|^{2}\right)^{1-\lambda \frac{k(1-\theta)}{2}},
$$

for $k$ defined in the assumptions and $\theta$ as in Lemma 4.

Then, calling $\mu=1-\lambda \frac{k(1-\theta)}{2}$, we obtain

$$
\frac{1}{2} \frac{d}{d t} \int_{\Omega}|\boldsymbol{h}(t)|^{2}+\int_{\Omega}|\nabla \times \boldsymbol{h}(t)|^{p}+C^{-\lambda}\left(\int_{\Omega}|\nabla \times \boldsymbol{h}(t)|^{p}\right)^{-\frac{\lambda k \theta}{p}}\left(\int_{\Omega}|\boldsymbol{h}(t)|^{2}\right)^{\mu} \leq 0 .
$$


So, denoting $y(t)=\int_{\Omega}|\boldsymbol{h}(t)|^{2}$ and $\Lambda(t)=2 C^{-\lambda}\left(\int_{\Omega}|\nabla \times \boldsymbol{h}(t)|^{p}\right)^{-\frac{\lambda k \theta}{p}}$, we arrive to the differential inequality

$$
y^{\prime}(t)+\Lambda(t) y(t)^{\mu} \leq 0 .
$$

Choosing $\beta=\frac{p}{\lambda k \theta}$, we get

$$
\int_{0}^{t} \Lambda(\tau)^{-\beta}=\left(2 C^{-\lambda}\right)^{-\beta} \int_{0}^{t} \int_{\Omega}|\nabla \times \boldsymbol{h}|^{p} \leq 2^{-(\beta+1)} C^{\lambda \beta}\left\|\boldsymbol{h}_{0}\right\|_{\boldsymbol{L}^{2}(\Omega)}^{2},
$$

because

$$
\frac{1}{2} \int_{\Omega}|\boldsymbol{h}(t)|^{2}+\int_{Q_{t}}|\nabla \times \boldsymbol{h}|^{p}+\int_{0}^{t}\left(\int_{\Omega}|\boldsymbol{h}|^{k}\right)^{-\lambda}\left(\int_{\Omega}|\boldsymbol{h}|^{2}\right)=\frac{1}{2} \int_{\Omega}\left|\boldsymbol{h}_{0}\right|^{2} .
$$

Denoting $2^{-(\beta+1)} C^{\lambda \beta}\left\|\boldsymbol{h}_{0}\right\|_{L^{2}(\Omega)}^{2}=C_{0}$ and using the reverse Hölder inequality with $0<r=\frac{\beta}{\beta+1}<1$ (so $\left.r^{\prime}=-\beta\right)$, we get

$$
\int_{0}^{t} \Lambda(\tau) \geq\left(\int_{0}^{t} 1\right)^{\frac{\beta+1}{\beta}}\left(\int_{0}^{t} \Lambda(\tau)^{-\beta}\right)^{-\frac{1}{\beta}} \geq C_{0}^{-\frac{1}{\beta}} t^{\frac{\beta+1}{\beta}} .
$$

Integrating (25),

$$
\frac{1}{1-\mu}\left(\int_{\Omega}|\boldsymbol{h}(t)|^{2}\right)^{1-\mu} \leq-C_{0}^{-\frac{1}{\beta}} t^{\frac{\beta+1}{\beta}}+\frac{1}{1-\mu}\left\|\boldsymbol{h}_{0}\right\|_{\boldsymbol{L}^{2}(\Omega)}^{2(1-\mu)} .
$$

So, defining $t_{*}$ by the relation

$$
t_{*}^{\frac{\beta+1}{\beta}}=\frac{C^{\lambda}}{(1-\mu) 2^{\frac{\beta+1}{\beta}}}\left\|\boldsymbol{h}_{0}\right\|_{\boldsymbol{L}^{2}(\Omega)}^{2-2 \mu+\frac{2}{\beta}},
$$

with $\mu$ and $\beta$ defined above and $C$ defined in (4), we have $\int_{\Omega}|\boldsymbol{h}(t)|^{2}=0$ for all $t \geq t_{*}$.

\section{Acknowledgments}

The first author was supported partially by the research project PTDC/MAT/ 110613/2009, FCT, Portugal.

The research of the second and third authors was partially supported by CMAT - "Centro de Matemática da Universidade do Minho", financed by FEDER Funds through "Programa Operacional Factores de Competitividade - COMPETE" and by Portuguese Funds through FCT - "Fundação para a Ciência e a Tecnologia", within the Project Est-C/MAT/UI0013/2011.

\section{References}

[1] Miranda, F., Rodrigues, J. F., and Santos, L. (2012) On a p-curl system arising in electromagnetism. Discrete Contin. Dyn. Syst. Ser. S, 5, 605-629.

[2] Antontsev, S. N., Díaz, J. I., and Shmarev, S. (2002) Energy methods for free boundary problems: Applications to nonlinear PDEs and fluid mechanics. Progress in Nonlinear Differential Equations and their Applications, 48, Birkhäuser Boston Inc.

[3] Antontsev, S. and Shmarev, S. (2009) Anisotropic parabolic equations with variable nonlinearity. Publ. Mat., 53, 355-399.

[4] Antontsev, S. N. and Shmarev, S. (2010) Blow-up of solutions to parabolic equations with nonstandard growth conditions. J. Comput. Appl. Math., 234, 2633-2645. 
[5] Antontsev, S. N. and Shmarev, S. (2010) Vanishing solutions of anisotropic parabolic equations with variable nonlinearity. J. Math. Anal. Appl., 361, 371-391.

[6] Antontsev, S. N. and Shmarev, S. (2008) Extinction of solutions of parabolic equations with variable anisotropic nonlinearities. Tr. Mat. Inst. Steklova, 261, 16-25.

[7] Miranda, F., Rodrigues, J. F., and Santos, L. (2009) A class of stationary nonlinear Maxwell systems. Math. Models Methods Appl. Sci., 19, 1883-1905.

[8] Amrouche, C. and Seloula, N. E. H. (2011) $L^{p}$-theory for vector potentials and Sobolev's inequalities for vector fields. C. R. Math. Acad. Sci. Paris, 349, 529-534.

[9] Simon, J. (1975) Quelques propriétés de solutions d'équations et d'inéquations d'évolution paraboliques non linéaires. Ann. Scuola Norm. Sup. Pisa Cl. Sci. (4), 2, 585-609.

[10] Simon, J. (1987) Compact sets in the space $L^{p}(0, T ; B)$. Ann. Mat. Pura Appl. (4), 146, 65-96.

[11] Lions, J.-L. (1969) Quelques méthodes de résolution des problèmes aux limites non linéaires. Dunod. 